\title{
Improved postural control after slackline training is accompanied by reduced H-reflexes
}

\author{
M. Keller ${ }^{1}$, J. Pfusterschmied ${ }^{2,3}$, M. Buchecker ${ }^{2,3}$, E. Müller ${ }^{2,3}$, W. Taube ${ }^{1}$ \\ ${ }^{1}$ Department of Medicine, Unit of Sports Science, University of Fribourg, Fribourg, Switzerland, ${ }^{2}$ Department Sport Science and \\ Kinesiology, University of Salzburg, Salzburg, Austria, ${ }^{3}$ Christian Doppler Laboratory "Biomechanics in Skiing", Salzburg, Austria \\ Corresponding author: Martin Keller, Department of Medicine, Unit Sports Science, University of Fribourg, Chemin du Musée \\ 3, CH-1700 Fribourg, Switzerland.Tel:+41 263007 286, Fax:+41 263009 771,E-mail: martin.keller@unifr.ch
}

\begin{abstract}
"Slacklining" represents a modern sports activity where people have to keep balance on a tightened ribbon. The first trials on the slackline result in uncontrollable lateral swing of the supporting leg. Training decreases those oscillations and therefore improves postural control. However, the underlying neural mechanisms are not known. Therefore, the present study aimed to highlight spinal adaptations going along with slackline training. Twenty-four subjects were either assigned to a training or a control group and postural control was assessed before and after the 10 training sessions. Additionally, soleus Hoffmann (H)reflexes were elicited to evaluate changes in the excitability
\end{abstract}

During the recent years more and more people became fascinated by the idea of balancing on tensioned nylon webbings, so-called "slacklines." Interestingly, slacklining found its way from a pure recreational sport into the training of elite athletes. In general, it is hard to get started and beginners can normally neither enter the line without assistance nor can they maintain balance. The first trials on the ribbon result in uncontrollable lateral swinging of the supporting leg and line. To our best knowledge, there is no publication covering the origin of this uncontrollable swinging. Therefore, it can only be speculated that the oscillations are caused by muscle stretch reflexes elicited by the fast deflection of the slackline to one side. The stretch-reflex activation may indeed counteract the initial deflection but probably results in an overshooting compensatory reaction to the other side so that the oscillation of leg and line builds-up to an uncontrollable sway. It may be speculated that experts have learned to suppress reflex-mediated joint oscillations.

The present study therefore aimed to clarify whether slackline training suppresses the spinal reflex activity. As it is difficult to assess and quantify stretch-reflex responses during activity, the electrically evoked Hoffmann (H)-reflex was used to eval- of the spinal reflex circuitry. Trained subjects were able to maintain balance on the slackline for at least $20 \mathrm{~s}$ $(P<0.001)$ and reduced platform movements on the balance board $(P<0.05)$. The $H$-reflexes were significantly diminished $(P<0.05)$ while no changes occurred in the background electromyography (bEMG). The control group showed no significant changes. From a functional point of view the reflex reduction may serve to suppress uncontrollable reflex mediated joint oscillations. As the bEMG remained unchanged, presynaptic rather than post-synaptic mechanisms are speculated to be responsible for the changes in the Ia-afferent transmission.

uate the Ia-afferent transmission before and after training. Previous studies have shown that the $\mathrm{H}$ reflex constitutes a sensitive measure of inferring spinal adaptations (for review see Zehr, 2002). With the focus on balancing, diminished H-reflexes were associated with increases in postural demands (Llewellyn et al., 1990; Earles et al., 2000; Taube et al., 2008b). Katz et al.(1988) demonstrated that this reduction largely relied on an enhanced presynaptic inhibition (PI) of Ia afferents. Longitudinal studies illustrated that balance training can further suppress the amplitude of the H-reflex and that this suppression goes along with improvements in postural control (Trimble \& Koceja, 1994; Taube et al., 2007a ,b; Gruber et al., 2007b).

In those "classical balance training studies" (BT), devices like half boards, wobbling boards, tilts, etc. were used to exercise postural control. One difference between classical BT and slacklining is therefore the movement of the training devices. In classical BT, all devices are more or less unvarying in their position in space, but show unstable characteristics. In contrast, slacklines move largely in space so that the supporting leg has to counteract lateral movements of the foot to balance the centre of gravity over the moving base of support. Hence, it seems obvious that classi- 
cal BT mostly activates the circumjacent ankle joint muscles, whereas slacklining activates muscle groups of ankle, knee and hip joints simultaneously.

The present study, therefore wanted to elucidate whether the adaptations after 4 weeks of slackline training are comparable to the adaptations reported after classical BT. It was hypothesized that slackline training results in diminished H-reflex excitability and improved postural control in different postural conditions. For this purpose, soleus (SOL) H-reflexes were evoked on the slackline and during different balance tasks before and after 4 weeks of slackline training. Furthermore, postural control was quantitatively assessed on a two-dimensional free-swinging platform to test the transferability of slackline training.

\section{Materials and methods}

\section{Subjects}

A total of 24 healthy subjects [training group (INT): six men, six women; control group (CON): six men, six women] without any history of neurologic or orthopedic disorders volunteered in the present study. All subjects gave their written consent before their inclusion to the study, which was conducted according to the latest version of the declaration of Helsinki and was approved by the local ethics commission. Subjects were included, if they had not taken part in BT and were unfamiliar with slacklining.

\section{Training intervention}

The INT conducted 4 weeks of slackline training with a total of 10 training sessions. The training sessions were performed on non-consecutive days. Two to three times a week, subjects of the INT trained for $90 \mathrm{~min}$ on $7-18 \mathrm{~m}$ long slacklines (width: $25.8 \mathrm{~mm}$; thickness: $2.49 \mathrm{~mm}$; fracture strain: $4.8 \%$ ). After every $2 \mathrm{~min}$ on the slackline, subjects had to rest for 2 min.

Subjects were asked to position the foot parallel on the line for all walking and standing exercises. However, in more advanced movements like turns the foot was also positioned perpendicular to the line. During the first three training sessions, subjects were taught to perform the basic movements (standing and walking forward) with assistance. With improvements of performance, assistance (bars and hands of coaches) was reduced until subjects were able to enter the line without help and could perform the first steps on their own. From the fourth training session the length of the line was gradually enhanced up to $18 \mathrm{~m}$ and the tension of the slackline was varied. Additionally, subjects were asked to perform more difficult movements (catch and pass a ball, stand up from a sitting position, turn on the line, juggling). A detailed description can be found in Table 1.

\section{General experimental overview}

Pre- and postmeasurements were conducted in the same laboratory with similar conditions (electric lighting, room temperature, time of day for testing, etc.). Subjects were tested 2-3 days before training started; posttesting was conducted 23 days after the last training session. After the preparation subjects conducted a short warm up (balance tasks of $5 \mathrm{~min}$ ) and were subsequently tested in different postural conditions.
Table 1. Description of the main aspects of each training session

\begin{tabular}{llc}
\hline $\begin{array}{l}\text { Session } \\
\text { Main aspects of } \\
\text { training sessions }\end{array}$ & $\begin{array}{l}\text { Length of the } \\
\text { line }(\mathrm{m})\end{array}$ \\
\hline 1 & Standing and first steps with support & $7-10$ \\
2 & Standing and first steps with minor support & $7-10$ \\
3 & Standing and steps without support & $7-10$ \\
4 & Walking forward and backward & $10-12$ \\
5 & Catch and pass a ball & $10-12$ \\
6 & Catch and pass a ball to the side & $10-12$ \\
7 & Turns on the line & $12-18$ \\
8 & Standing up from a sitting position & $12-18$ \\
9 & Juggling & $12-18$ \\
10 & Two people on one line & $12-18$ \\
\hline
\end{tabular}

The postural demands were enhanced with improvements in postural control.

To exclude fatigue and learning effects during the measurements, the chronological order of the testing conditions was randomized.

\section{Balance tasks}

Dynamic postural stability was evaluated using a multi-axial free-swinging platform (Posturomed ${ }^{\mathrm{TM}}$, Haider Bioswing, Pullenreuth, Germany). The quadratic platform was mounted on four pendulums to guarantee free movements in all directions of the transversal plane. The device could be moved out of the middle position and was magnetically fixed in a deflected position with a displacement of approximately $25 \mathrm{~mm}$. To test postural control of the subjects, a medio-lateral (ml) perturbation impulse was unexpectedly applied by releasing the magnet. When the fixation was released, translational perturbations in the $\mathrm{ml}$ direction were produced (for technical details see Muller et al., 2004). Joystick potentiometers connected to the platform measured the platform movements in anteriorposterior (ap) and $\mathrm{ml}$ direction. For both directions platform movements were measured in centimeters. Subjects were asked to stabilize platform movements as quickly as possible in both $\mathrm{ml}$ and ap direction. To minimize the assessment of learning effects, a 2-min trial was given to accustom the subjects to the device. To get familiar with the perturbation task, five perturbations were executed before the two test trials were recorded. Subjects were asked to reduce the platform movements during the initial $15 \mathrm{~s}$ after the perturbation while standing on the device with their right leg slightly bent $\left(\sim 30^{\circ}\right)$ and hands held akimbo. During the assessment of postural control no electrical stimuli were applied.

To test the slackline performance, subjects were asked to balance with the foot parallel on the slackline for $20 \mathrm{~s}$. The tension of the slackline was adjusted to $1000 \pm 5 \mathrm{~N}$ in the preand postmeasurement. Each subject started in the middle of the slackline and was given three trials to accomplish the task. Because no subject was able to enter the line without support in the pre-test, subjects were assisted to find a starting position. When subjects gave a "go signal" support was pulled away and a countdown clock was started. If subjects touched the ground before the $20 \mathrm{~s}$ limit, the trial was counted as "not succeeded." Data were collected on a nominal scale level (succeeded vs not succeeded).

\section{Electromyographic (EMG) recordings}

To monitor muscle activity in the present study, EMG activity was recorded from the SOL, gastrocnemius medialis (GM) 
and tibialis anterior (TA) muscles. After skin preparation with sand paper and disinfectant spray, surface electrodes (Hellige ${ }^{\mathbb{R}}$, Freiburg, Germany; diameter $10 \mathrm{~mm}$, center to center distance $2 \mathrm{~cm}$ ) were attached to the skin for bipolar EMG recording. The interelectrode resistance was kept below $5 \mathrm{k} \Omega$. The positioning of the electrodes was in line with the underlying longitudinal muscle fibers. The reference electrode was attached to the tibial shaft. The EMG was recorded with a sampling rate of $4 \mathrm{kHz}$, amplified $(\times 1000)$ and bandpass-filtered $(10-1000 \mathrm{~Hz})$. During the measurement, the raw EMG could be observed online and was stored on a hard drive for later analysis with custom-built software (LabView ${ }^{\mathbb{B}}$ based National Instruments ${ }^{\mathbb{B}}$, Austin, Texas, USA). balancing on the slackline. For all subjects, balancing on the slackline without assistance was not possible in the premeasurement. After training, all subjects could indeed maintain balance on the slackline without external support but were not able to counterbalance perturbations evoked by the peripheral nerve stimulation. Hence, subjects were allowed in pre- and postmeasurements to gently grip for a finger of one of the authors in order to maintain balance. Previously it was shown that different levels of lower limb joint loading can alter the $\mathrm{H}$ reflex (Nakazawa et al., 2004) as well as the muscle length (Hwang, 2002). Therefore, care was taken that subjects started with similar joint angles in all conditions and that limb loading did not change between the instable conditions.

\section{Peripheral nerve stimulation}

An electrical stimulator (AS 100, Alea Solutions, Zürich, Switzerland) was used for electrical stimulation. Single square-wave pulses with duration of $1 \mathrm{~ms}$ were applied to elicit the posterior tibial nerve. For this purpose the cathode $(2 \mathrm{~cm}$ in diameter) was placed in the popliteal fossa and moved until the best position was found for eliciting a muscular response in SOL. The anode $(10 \times 5 \mathrm{~cm}$ dispersal pad $)$ was fixed directly below the patella on the anterior aspect of the knee. As the tibial nerve contains Ia afferent as well as efferent pathways, two muscular responses with different delays can be obtained in the EMG. The $M$-wave with a delay of $\sim 10 \mathrm{~ms}$ is mediated directly to the neuromuscular junction by the efferent motor axons, whereas the H-reflex with a delay of $\sim 35 \mathrm{~ms}$ travels from the point of stimulation via Ia afferents to the $\alpha$ motoneurons and from there on to the neuromuscular junction. As the diameter of Ia afferents is larger compared with motor axons, the former are recruited with smaller stimulus intensities. When increasing the stimulus intensity from a low level, where no muscular response can be obtained, to a medium level, which is sufficient to excite the H-reflex, and subsequently to high-level stimulus intensities, which is capable to elicit the maximum $M$-wave, an $H / M$-recruitment curve can be obtained. Expressing $H_{\max }$ in relation to $M_{\max }(H / M$-ratio) counteracts the potential problem of non-identical EMG recording qualities in pre- and postmeasurement (Aagaard et al., 2002). The H-reflex was first applied during bipedal quiet stance on solid ground to obtain a reference $H / M$ ratio. To create an $H / M$ recruitment curve 20-30 trials were collected with an interstimulus interval of $4 \mathrm{~s}$ (Fig. 1). Additionally, recruitment curves were recorded when performing unperturbed one leg stance with the knee slightly bent on the Posturomed, when balancing on an air cushion and when

\section{Data analysis and statistics}

$\mathrm{H}$-reflexes and $M$-waves were expressed as peak-to-peak amplitudes. For the calculation of the $H_{\max } / M_{\max }$ ratios ( $=H / M$ ratios $)$ the maximum $\mathrm{H}$-reflex and the maximum $M$-wave were determined in each condition. Muscular activity of SOL, TA and GM was quantified by means of root mean square values. The time-frame for background EMG (bEMG) analysis was defined from 1.5 to $3.5 \mathrm{~s}$ after each stimulus due to four reasons: first, it was considered to be important to assess the muscular activity during the same trial as the electrophysiological data; second, the disturbing influence of the electric stimulation should be excluded; third, the time interval should be long enough to display representative muscle activity and fourth, potential preparation effects preceding each stimulus should be excluded. In later analysis no preparation effects were found in bEMG (results not shown). For normalization and comparability of pre- and postmeasurements, muscular activity of SOL was expressed to the corresponding $M_{\max }$ value. One subject of the INT was excluded of the H-reflex and EMG analysis as it was not possible to evoke H-reflex responses.

The effect of the intervention on the $H / M$ ratios was analyzed by applying a repeated measures analysis of variance (ANOVA) procedure with the factors of time (pre vs post), task (bipedal stance vs Posturomed vs cushion vs slackline) and group (control vs intervention). For quantification of differences within a group a repeated measures ANOVA procedure with the factors of task (bipedal stance vs posturomed vs cushion vs slackline) and time (pre vs post) was conducted.

Differences in muscular activity were analyzed with a repeated measures ANOVA procedure $[2$ (time) $\times 4$ $($ task $) \times 2$ (group) $\times 3$ (muscle) $]$. The bEMG of the $\mathrm{SOL}$
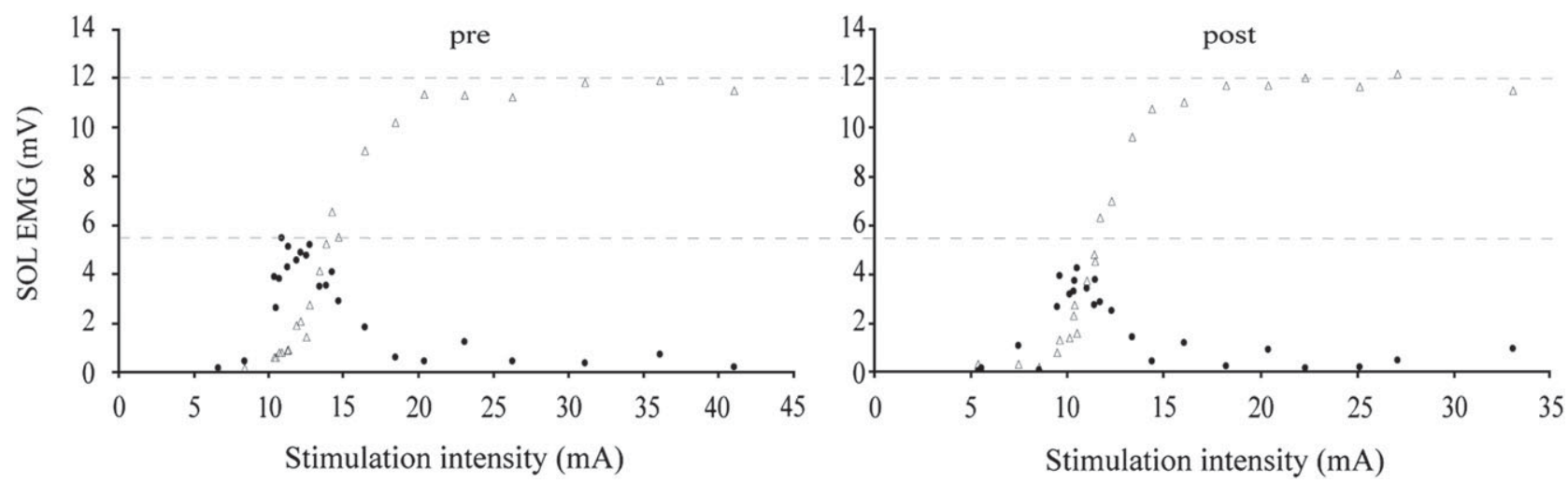

Fig. 1. Two exemplary H-reflex recruitment curves ( $\bullet$ : H-reflexes; $\Delta$ : M-waves) from one trained subject before and after the training period. These data were recorded when balancing on the slackline. It is clearly illustrated that the $\mathrm{H}^{\text {max }} / \mathrm{M}^{\text {max }}$-ratio is reduced after training. 
was additionally normalized to $M_{\max }$ and was statistically analyzed in the same way.

To reveal differences in platform movements of the Posturomed, a repeated measures ANOVA was conducted [2 (time) $\times 2$ (group) $\times 3$ (direction) $]$. In case of significant $F$ values $(P<0.05)$, changes with respect to the platform movement direction were compared by Bonferroni's corrected paired two-sided tests (Student's $t$-test). Fisher's exact test was used to assess changes of postural stability in the slackline condition. For all tests the significance level was set to $P \leq 0.05$. SPSS 16.0 was used for statistical analysis. All values are presented as means with standard deviation.

\section{Results}

\section{Balance parameters}

Before training, none of the subjects was able to maintain balance for $20 \mathrm{~s}$ on the slackline in any of the three trials. After training, all subjects participating in the intervention group could balance without assistance on the slackline for at least $20 \mathrm{~s}$. Most of the trained subjects $(n=11)$ succeeded on the first trial whereas one subject needed a second trial. None of the subjects of the CON could balance for $20 \mathrm{~s}$ on the slackline in the post-test. Hence, the INT significantly improved performance compared with the CON $(P<0.0001)$.

The tests of postural control using the Posturomed resulted in a significant difference over time between training and control group (time $\times$ group: $\left.F_{1,22}=5.85 ; P<0.05\right)$. The INT showed significant changes (time: $F=17.88 ; P=0.01$; ap direction: pre:
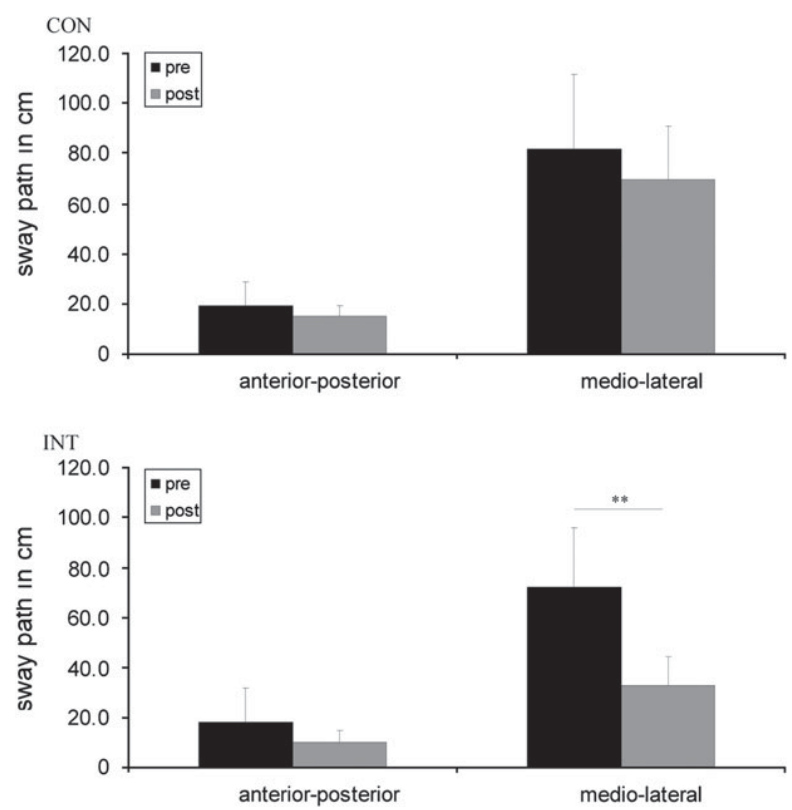

Fig. 2. Sway path displayed for anterior-posterior and medio-lateral direction. Further analysis showed adaptations over time for the training, but not for the control group. Highly significant adaptations were found in mediolateral direction whereas changes in the anterior-posterior direction were not significant. ${ }^{*} P<0.01$.
$18.41 \pm 13.61 \mathrm{~cm}$ vs post: $10.08 \pm 4.96 \mathrm{~cm}$; change: $83 \%$; ml direction: pre: $82.29 \pm 42.00 \mathrm{~cm}$ vs post: $34.94 \pm 13.52 \mathrm{~cm}$; change: $136 \%$ ) whereas no changes were found for the CON (time: $F=2.72$; $P=0.13$; ap direction: pre: $19.16 \pm 9.32 \mathrm{~cm}$ vs post: $15.12 \pm 3.93 \mathrm{~cm}$; change: $27 \%$; ml direction: pre: $81.64 \pm 28.48 \mathrm{~cm}$ vs post: $69.61 \pm 20.47 \mathrm{~cm}$; change: $17 \%$ ) (Fig. 2).

\section{Reflex size and EMG recordings}

Reflex recordings were statistically different in preand post-test with respect to the group (time group: $\left.F_{1,21}=7.22 ; P<0.05\right)$. However, only the INT demonstrated significantly reduced H-reflexes in the post measurement (time: $F_{1,10}=11.99$; $P<0.01 ; H / M$ ratios: stance condition: pre: $0.50 \pm 0.14$ vs post: $0.38 \pm 0.12$; change: $31 \%$; cushion: pre: $0.56 \pm 0.13$ vs post: $0.40 \pm 0.20$; change: $40 \%$; posturomed: pre: $0.47 \pm 0.15$ vs post: $0.34 \pm 0.19$; change: $39 \%$; slackline: pre: $0.49 \pm 0.18$ vs post: $0.38 \pm 0.15$; change: $29 \%$ ) whereas the values remained unchanged in the CON (time: $F_{1,11}=1.12 ; \quad P=0.31 ; H / M$ ratios: stance condition: pre: $0.51 \pm 0.20$ vs post: $0.53 \pm 0.18$; change: $2 \%$; cushion: pre: $0.56 \pm 0.18$ vs post: $0.52 \pm 0.18$; change: $7 \%$; posturomed: pre: $0.53 \pm 0.20$ vs post: $0.49 \pm 0.15$; change: $8 \%$; slackline: pre: $0.53 \pm 0.17$ vs post: $0.51 \pm 0.18$; change: $4 \%$ ). There was no interaction of time and condition for the H-reflexes in any group (time $\times$ condition:
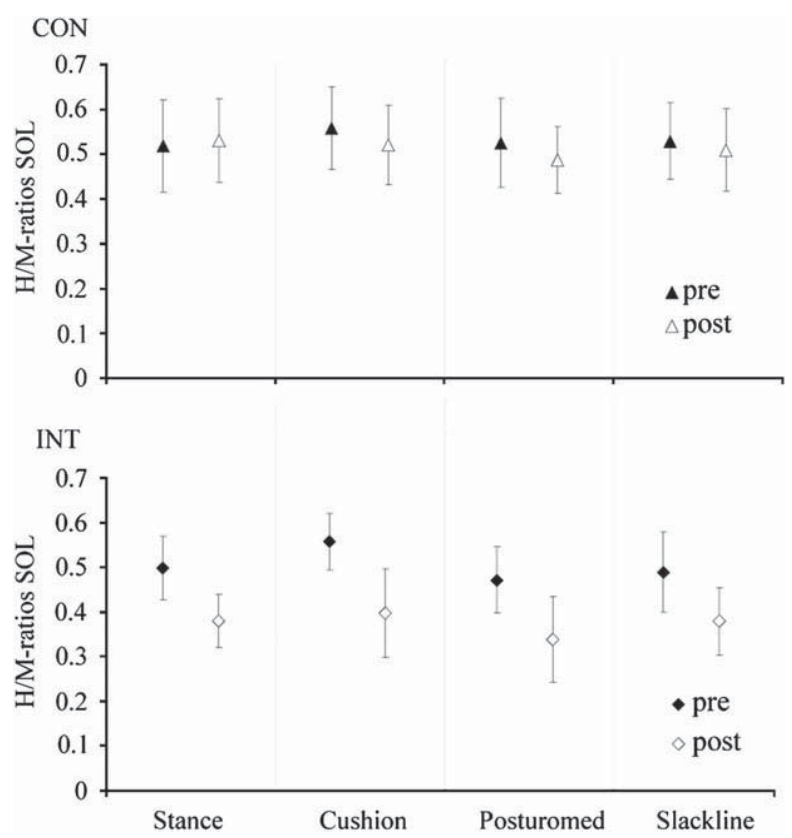

Fig. 3. H/M-ratios are displayed for training (pre: ; post: $\diamond$ ) and control (pre: $\boldsymbol{\Delta}$; post: $\Delta$ ) group. In all conditions a distinct decrease in reflex activity can be seen after the slackline training. No changes occurred in the control group. 
INT: $\quad F_{1,10}=0.35 ; \quad P>0.05 ; \quad$ CON: $\quad F_{1,11}=0.22$; $P>0.05)$ (Fig. 3).

Non-normalized as well as normalized muscular activity showed no differences after the training period in any group (non-normalized: time $\times$ group: $F_{1,21}=0.39 ; \quad P=0.54 ;$ normalized: time $\times$ group: $F_{1,21}=1.92 ; P=0.18$; not illustrated).

\section{Discussion}

The main finding of the present study was that 4 weeks of slackline training improved postural control and was accompanied by reductions of the H-reflex. Slackline training therefore seems to induce similar neuromuscular adaptations as classical BT. It is speculated that the reduction of the H-reflex may serve to inhibit reflex mediated joint oscillations, responsible for uncontrollable sway.

\section{Adaptations in postural control}

After slackline training, each subject of the INT could balance at least for $20 \mathrm{~s}$ on the ribbon. Moreover, most subjects were able to walk, turn and perform dual-tasks on the slackline after training. Thus, although slacklining was experienced highly challenging at the beginning of the training, subjects with ordinary balance skills were able to learn this task within 4 weeks.

To clarify whether improvements in postural control after slackline training were specific to the slackline condition or could be transferred to other balance tasks, postural control was not only assessed on the slackline but also on a free-swinging platform (Posturomed). Interestingly, the most pronounced reduction in platform movements of the Posturomed could be detected in the $\mathrm{ml}$ direction. This seems reasonable as walking and standing on a slackline results in mostly $\mathrm{ml}$ oscillations. Therefore, postural adaptations in the present study are more pronounced in balance tasks closely related to the training task. More importantly, this finding proposes that training-related improvements are not restricted to slacklining but may be transferred to other challenging balance tasks (e.g. on the Posturomed). Such a transfer of acquired postural skills to other, not especially learned balance tasks, was also reported after classical BT (Granacher et al., 2006; Taube et al., 2007a) and in-line training (Taube et al., 2010).

\section{Adaptations of the SOL H-reflex}

The present study demonstrated that 4 weeks of slackline training resulted in diminished $H / M$ ratios on the slackline but also during bipedal stance on solid ground and while balancing on a cushion and the Posturomed. These results are in line with the observation of reduced H-reflexes after classical BT (Taube et al., 2007a, b; Gruber et al., 2007a). In those previous studies, the suppression of the Ia-afferent transmission was argued to be important to inhibit reflex mediated joint oscillations (for review see Taube et al., 2008a). The unwanted and uncontrollable joint oscillations are assumed to origin from muscle stretch reflexes that occur when a fast deflection of the slackline or a fast tilt of a classical BT device takes place and the counteracting stretch reflex results in an overshooting joint repositioning. This overcorrection may trigger subsequent stretch reflexes that are probably responsible for the build up of the described joint oscillations. Suppression of the Ia-afferent excitation to the $\alpha$-motoneuron pool could therefore indeed attenuate destabilizing joint movements.

In the present study the SOL H-reflex was analyzed. At the same time it is obvious that the highest movement amplitudes of the slackline occurred in the $\mathrm{ml}$ direction. Therefore, one may argue that the SOL muscle has minor potential for stabilizing the joint in $\mathrm{ml}$ direction and other muscles are more important for this task. This is in fact true and was considered beforehand, but for reasons of comparability to classical BT and due to the high reliability of this measurement in SOL it was decided to measure the SOL H-reflex. Nevertheless, the potential for reflex modulation on muscles that work in $\mathrm{ml}$ direction may be even higher.

\section{Potential mechanisms responsible for the reduction of the SOL H-reflex}

As nicely summarized by Schieppati (1987) neural mechanisms can influence the amplitude of the $\mathrm{H}$ reflex. One candidate for reflex modulation is an altered level of the bEMG and thus the excitability of the motoneuronpool of the agonistic muscle(s) (Schieppati, 1987). However, as SOL and GM activity and the related activity from SOL to $M_{\max }$ remained unchanged after training, this cannot explain the observed changes in the H-reflex amplitude in the present study. A second potential candidate for reflex modulation is reciprocal inhibition (Zehr, 2002): Reciprocal inhibition refers to a relative decrease in agonistic motoneuron activity with an increasing activity of the antagonistic muscle and was demonstrated to be present in both extensors and flexors in man (Crone et al., 1987). However, as the muscular activity of the TA remained unchanged after training, reciprocal inhibition can also not account for the present findings. Closely related to the previous point is the level of co-contraction: the magnitude of co-contraction of TA and SOL was shown to be negatively correlated with the amplitude 
of the SOL H-reflex (Perez et al., 2007). However, an enhanced co-contraction of plantar and dorsiflexors was not observed after training.

One mechanism that could explain the present results is PI. PI was previously shown to influence reflex propagation (Capaday \& Stein, 1987; Yang \& Whelan, 1993) via supraspinal pathways (Hultborn et al., 1987; Oishi et al., 1994; Nielsen et al., 1995). Moreover, based on the results of Katz et al. (1988) showing an increased level of PI with increases in the postural demand, it can be speculated that modulation of PI plays an important role in adjusting the neuromuscular system to unstable support conditions. Furthermore, training may be beneficial in adapting the modulation of PI as was proposed after strength (Aagaard et al., 2002) and BT (Trimble \& Koceja, 1994; Gruber et al., 2007b; Taube et al., 2007a,b). In line with these studies it could be assumed that PI is a likely mechanism responsible for altering reflex behavior in the present study. However, it has to be mentioned that the present methodology cannot exclude modulation of the H-reflex due to activity in other peripheral afferent pathways originating from receptors like Golgi tendon organs or cutaneous mechano receptors. Furthermore, previously it was shown that position and loading of knee and hip joint may influence the SOL H-reflex (Tanabe et al., 2004; Knikou 2006; Kamiya et al., 2006). The activity of the knee extensors may have also affected the H-reflex by modulating the extent of recurrent inhibition (Lamy et al., 2008). Thus, slackline training could have potentially altered motor performance and as a consequence the H-reflex was altered rather than changes in the H-reflex led to functional improvements in balance performance. As it is impossible to monitor all pathways acting on the H-reflex, Zehr (2002) emphasized the need for exclusion of extraneous factors which might alter the H-reflex. Therefore, measurement conditions in pre- and posttest were kept constant and were in line with recommendations for posturo- graphic testing in the present study (Kapteyn et al., 1983).

\section{Perspectives}

This is the first study that shows that slacklinetraining alters postural control and Ia-afferent transmission (H-reflexes) similar to a classical BT. Although not tested in this study it might be assumed that slackline-training and classical BT may have more things in common than improving postural control and altering the reflex behavior. Classical BT was shown to improve motor performance in rapid isometric contractions (Gruber et al., 2007a) and to ameliorate jumping abilities (Taube et al., 2007b). Additionally, prospective studies demonstrated preventive aspects of classical BT in different team sports with respect to knee injuries (Myklebust et al., 2003) and ankle sprains (McGuine \& Keene, 2006), improved regeneration of neuromuscular structures following injury (Henriksson et al., 2001) and a diminished injury re-occurrence (Holme et al., 1999). However, despite the many beneficial aspects of classical BT, it is rarely an inherent part of training, as athletes are often not motivated to participate in classical BT due to its uniformity. Slacklining on the other hand can be classified as a funsport activity and may be more joyful and even more demanding in some aspects as a classical BT. Thus, many athletes are highly motivated in trying to improve their balance performance on the slackline without knowing anything about the potential positive side effects described above. To scientifically justify slacklining as an intervention to improve motor performance (apart from balance), rehabilitation and prevention, further research is needed to uncover the physiological benefits and risks associated with this kind of sports activity.

Key words: balance, sensorimotor training, peripheral nerve stimulation.

\section{References}

Aagaard P, Simonsen EB, Andersen JL, Magnusson P, Dyhre-Poulsen P. Neural adaptation to resistance training: changes in evoked V-wave and H-reflex responses. J Appl Physiol 2002: 92: 2309-2318.

Capaday C, Stein RB. Difference in the amplitude of the human soleus $\mathrm{H}$ reflex during walking and running. J Physiol 1987: 392: 513-522.
Crone C, Hultborn H, Jespersen B, Nielsen J. Reciprocal Ia inhibition between ankle flexors and extensors in man. J Physiol 1987: 389: 163-185.

Earles DR, Koceja DM, Shively CW. Environmental changes in soleus $\mathrm{H}$ reflex excitability in young and elderly subjects. Int J Neurosci 2000: 105: $1-13$.

Granacher U, Gollhofer A, Strass D. Training induced adaptations in characteristics of postural reflexes in elderly men. Gait Posture 2006: 24: 459-466.

Gruber M, Gruber SB, Taube W, Schubert M, Beck SC, Gollhofer A. Differential effects of ballistic versus sensorimotor training on rate of force development and neural activation in humans. J Strength Cond Res 2007a: 21: 274-282.

Gruber M, Taube W, Gollhofer A, Beck S, Amtage F, Schubert M. Trainingspecific adaptations of $\mathrm{H}$ - and stretch 
reflexes in human soleus muscle. J Mot Behav 2007b: 39: 68-78.

Henriksson M, Ledin T, Good L.

Postural control after anterior cruciate ligament reconstruction and functional rehabilitation. Am J Sports Med 2001: 29: 359-366.

Holme E, Magnusson SP, Becher K, Bieler T, Aagaard P, Kjaer M. The effect of supervised rehabilitation on strength, postural sway, position sense and re-injury risk after acute ankle ligament sprain. Scand J Med Sci Sports 1999: 9: 104-109.

Hultborn H, Meunier S, PierrotDeseilligny E, Shindo M. Changes in presynaptic inhibition of Ia fibres at the onset of voluntary contraction in man. J Physiol 1987: 389: 757-772.

Hwang IS. Assessment of soleus motoneuronal excitability using the joint angle dependent $\mathrm{H}$ reflex in humans. J Electromyogr Kinesiol 2002: 12: 361-366.

Kamiya A, Tanabe S, Muraoka Y, Masakado Y. Modulation of the soleus H-reflex during static and dynamic imposed hip angle changes. Int J Neurosci 2006: 116: 1045-1053.

Kapteyn TS, Bles W, Njiokiktjien CJ, Kodde L, Massen CH, Mol JM. Standardization in platform stabilometry being a part of posturography. Agressologie 1983: 24: 321-326.

Katz R, Meunier S, Pierrot-Deseilligny E. Changes in presynaptic inhibition of Ia fibres in man while standing. Brain 1988: 111(Part 2): 417-437.

Knikou M. Effects of changes in hip position on actions of spinal inhibitory interneurons in humans. Int J Neurosci 2006: 116: 945-961.

Lamy JC, Iglesias C, Lackmy A., Nielsen JB., Katz R., Marchand-Pauvert V. Modulation of recurrent inhibition from knee extensors to ankle motoneurones during human walking. J Physiol 2008: 586: 59315946.

Llewellyn M, Yang JF, Prochazka A. Human $\mathrm{H}$-reflexes are smaller in difficult beam walking than in normal treadmill walking. Exp Brain Res 1990: 83: 22-28.

McGuine TA, Keene JS. The effect of a balance training program on the risk of ankle sprains in high school athletes. Am J Sports Med 2006: 34: 1103-1111.

Muller O, Gunther M, Krauss I, Horstmann T. Physical characterization of the therapeutic device Posturomed as a measuring device - presentation of a procedure to characterize balancing ability. Biomed Tech (Berlin) 2004: 49: 56-60.

Myklebust G, Engebretsen L, Braekken IH, Skjolberg A, Olsen OE, Bahr R. Prevention of anterior cruciate ligament injuries in female team handball players: a prospective intervention study over three seasons. Clin J Sport Med 2003: 13: 71-78.

Nakazawa K, Miyoshi T, Sekiguchi H, Nozaki D, Akai M, Yano H. Effects of loading and unloading of lower limb joints on the soleus H-reflex in standing humans. Clin Neurophysiol 2004: 115: 1296-1304.

Nielsen J, Crone C, Sinkjaer T, Toft E, Hultborn H. Central control of reciprocal inhibition during fictive dorsiflexion in man. Exp Brain Res 1995: 104: 99-106.

Oishi K, Kimura M, Yasukawa M, Yoneda T, Maeshima T. Amplitude reduction of $\mathrm{H}$-reflex during mental movement simulation in elite athletes. Behav Brain Res 1994: 62: 55-61.

Perez MA, Lundbye-Jensen J, Nielsen JB. Task-specific depression of the soleus $\mathrm{H}$-reflex after cocontraction training of antagonistic ankle muscles. J Neurophysiol 2007: 98: 3677-3687.

Schieppati M. The Hoffmann reflex: a means of assessing spinal reflex excitability and its descending control in man. Prog Neurobiol 1987: 28: 345376.

Tanabe S, Muraoka Y, Kamiya A, Tomita Y, Masakado Y. Passive movement of hip and knee joints decreases the amplitude of soleus $\mathrm{H}$ reflex in stroke patients. Electromyogr Clin Neurophysiol 2004: 44: 365-370.

Taube W, Bracht D, Besemer C, Gollhofer A. The effect of inline skating on postural control in elderly people. Deutsche Zeitschrift für Sportmedizin 2010: 2: 45-51.

Taube W, Gruber M, Beck S, Faist M, Gollhofer A, Schubert M. Cortical and spinal adaptations induced by balance training: correlation between stance stability and corticospinal activation. Acta Physiol (Oxf) 2007a: 189: 347358.

Taube W, Gruber M, Gollhofer A. Spinal and supraspinal adaptations associated with balance training and their functional relevance. Acta Physiol (Oxf) 2008a: 193: 101-116.

Taube W, Kullmann N, Leukel C, Kurz O, Amtage F, Gollhofer A. Differential reflex adaptations following sensorimotor and strength training in young elite athletes. Int J Sports Med 2007b: 28: 999-1005.

Taube W, Leukel C, Gollhofer A. Influence of enhanced visual feedback on postural control and spinal reflex modulation during stance. Exp Brain Res 2008b: 188: 353-361.

Trimble MH, Koceja DM. Modulation of the triceps surae H-reflex with training. Int J Neurosci 1994: 76: 293-303.

Yang JF, Whelan PJ. Neural mechanisms that contribute to cyclical modulation of the soleus H-reflex in walking in humans. Exp Brain Res 1993: 95: 547556.

Zehr EP. Considerations for use of the Hoffmann reflex in exercise studies. Eur J Appl Physiol 2002: 86: 455-468. 